\title{
A NUMERICAL BOUNDARY INTEGRAL EQUATION METHOD FOR ELASTODYNAMICS. I
}

\author{
By David M. Cole, Dan D. Kosloff, and J. Bernard Minster
}

\section{ABSTRACT}

The boundary initial value problems of elastodynamics are formulated as boundary integral equations. It is shown that these integral equations may be solved by time-stepping numerical methods for the unknown boundary values. A specific numerical scheme is presented for antiplane strain problems and a numerical example is given.

\section{INTRODUCTION}

Many of the boundary value problems in geophysics and engineering involve bodies whose geometries preclude the construction of analytical solutions. In such cases it is often necessary to resort to numerical techniques. The most commonly used numerical methods, finite differences and finite elements, require a computational grid which fills the solution domain and is fine enough to resolve the features of interest in the solution. In large domains this places strong constraints on the resolution of solutions which may be achieved in practice.

Many problems of interest involve the solution of a system of linear partial differential equations for which a surface integral representation exists. In this form the solution at an interior point of a homogeneous body is written as a convolution of the physically relevant boundary values, initial values, and interior sources with a singular solution of the system of differential equations. In general, the boundary values will not all be known and unless the singular solution used coincides with the Green's tensor for the problem such an integral representation is merely another statement of the problem

Through a limiting process these integral representations may be converted into integral equations involving only the boundary values, initial values, and interior sources. This integral equation form is an equally valid statement of the problem and has the advantage that the only unknown quantities are boundary values, since the interior sources and initial values are usually specified. The boundary value problem is formulated directly in terms of boundary values and the solution at interior points need not be considered, although it may be evaluated at these points directly from the integral representation, once the boundary integral equations (BIE) have been solved. In many geophysical modeling problems this is not necessary, since physical observations are often confined to boundaries.

This paper directs its attention to the reduction of the BIE for linear elastodynamics to general purpose algebraic relations among the boundary values at a finite set of space and time points. These algebraic relations can then be used in a timestepping algorithm to obtain approximate solutions for any boundary value-initial value problem involving the elastic body for which they were formulated.

The advantages of the BIE method lie primarily in the reduction of the number of spatial dimensions of the problem by one and by formulating the problem directly in terms of the boundary values. Since one discretizes only the boundary of the body the number of unknown variables in the numerical problem is appropriately reduced and the problem of creating acceptable space filling three-dimensional grids is eliminated. In addition, an infinite homogeneous exterior domain may be included 
through a BIE on its interior surface; this is a "transparent" boundary condition. Infinite boundaries must still be truncated unless suitably vanishing Green's tensors are available.

Numerical BIE methods have been successfully applied to problems resulting from elliptic and parabolic type partial differential equations. These applications include problems from elastostatics, time harmonic elastodynamics, elastoplasticity, heat flow, electromagnetic scattering, and others. A representative collection of papers may be found in Cruse and Rizzo (1975). The BIE approach is generally found to be competitive with volume gridding methods for problems to which both are applicable. A few problems arising from hyperbolic differential equations have also been treated. Das (1976) and Das and Aki (1977) solve an elstodynamic BIE on a line in two-dimensional fracture dynamics problems. Mitzner (1967) and Friedman and Shaw (1962) treat two-dimensional problems with special boundary conditions. These studies use a time-stepping approach but do not consider a sufficiently general problem to be widely useful. Transient elastic wave propagation problems have been treated by Cruse (1968), solving elliptic BIE problems in the Laplace transform domain and inverting the Laplace transform to obtain time-domain solutions. This approach does not take advantage of the causal properties of the time dependent Green's tensors and requires the solution of a full matrix equation for each value of the transform variable. The time-domain approach taken here permits the development of an explicit stepping scheme and hence greater economy in the calculation of numerical solutions.

The remainder of this paper consists of three sections. The second section contains a derivation of the time dependent displacement BIE for elastodynamics. In the third section a discretization scheme for the BIE is developed in the context of interpolation functions and a specific interpolation method is presented for antiplane strain problems. In the fourth section the results of a simple antiplane strain problem are presented. An accompanying paper (Cole, 1979, in preparation), will present extensive numerical calculations and a discussion of the performance of the technique.

\section{Analytic Boundary Integral Equations}

The equations of motion of isotropic linearized elasticity are

$$
\begin{gathered}
\rho \frac{\partial^{2}}{\partial t^{2}} u_{i}=\sigma_{i j, j}+f_{i} \\
\sigma_{i j}=C_{i j k l} u_{k, l} \\
C_{i j k l}=\lambda \delta_{i j} \delta_{k l}+\mu\left(\delta_{i k} \delta_{j l}+\delta_{i l} \delta_{j k}\right) .
\end{gathered}
$$

Here $\lambda$ and $\mu$ are the Lame' constants, $\rho$ is the density, and $\sigma_{i j}$ are the cartesian stress components; $\delta_{i j}$ is the Kronecker delta and summation over repeated indices is assumed unless otherwise indicated; $f$ is the body force density and $u_{k, l}=\partial / \partial_{x l u k}$.

Consider the motion of a homogeneous body $B$ with interior $B^{\prime}$ and piecewise Liapunov boundary $\partial B$ (e.g., Kupradze, 1963) with normal $\mathbf{n}$ for times $t \geqq 0$. The body forces $\mathbf{f}$ and initial values, $\mathbf{u}(\mathbf{r}, t)$ and $\partial / \partial t \mathbf{u}(\mathbf{r}, t)$ at $t=0$, are specified for $\mathbf{r} \in B$. In addition there will be conditions which the boundary values, $\mathbf{u}(\mathbf{r}, t)$ and $\tau(\mathbf{r}, t)=\boldsymbol{\sigma} \cdot \mathbf{n}$ at $\mathbf{r} \in \partial B$, must satisfy. (For a well-posed problem these conditions will not specify $\mathbf{u}$ and $\tau$ completely.) 
A fundamental solution $\mathscr{G}\left(\mathbf{r}, t ; \mathbf{r}_{0}, t_{0}\right)$ of equation (1) is defined by

$$
\rho \frac{\partial^{2}}{\partial t_{0}^{2}} G_{i j}=C_{i p q s} G_{q j, s_{0} p_{0}}+\delta_{i j} \delta\left(t-t_{0}\right) \delta\left(r-r_{0}\right)
$$

for $\mathbf{r}, \mathbf{r}_{0} \in B^{\prime}$, where $f_{s_{0}}$ denotes differentiation with respect to the $s$-component of $\mathbf{r}_{0}$, the source coordinate. $\delta(t)$ is the Dirac delta function.

$\mathscr{G}$ also has the causality property

$$
G_{i j}\left(\mathbf{r}, t ; \mathbf{r}_{0}, t_{0}\right) \equiv 0, \quad \alpha\left(t-t_{0}\right)<\left|\mathbf{r}-\mathbf{r}_{0}\right|
$$

and the time translation property

$$
G_{i j}\left(\mathbf{r}, t+t_{1} ; \mathbf{r}_{0}, t_{0}+t_{1}\right)=G_{i j}\left(\mathbf{r}, t ; \mathbf{r}_{0}, t_{0}\right)
$$

where $\alpha=[(\lambda+2 \mu) / \rho]^{1 / 2}$ and $\beta=(\mu / \rho)^{1 / 2}$ are the compressional and shear-wave velocities, respectively.

From equations (1) and (3) one may obtain [see e.g., de Hoop (1958); Archambeau and Minster (1978). Wheeler and Sternberg (1968) give a precise treatment].

$$
\begin{aligned}
\mathbf{u}(\mathbf{r}, t)= & \int_{t_{0}=0}^{\infty} \oint_{\partial B}\left\{\mathscr{G} \cdot \tau\left(\mathbf{r}_{0}, t_{0}\right)-\mathbf{u}\left(\mathbf{r}_{0}, t_{0}\right) \cdot \mathbf{K} \cdot \mathbf{n}\right\} d S_{0} d t_{0} \\
& +\left.\int_{B} \rho\left\{\mathscr{G} \cdot \frac{\partial}{\partial t_{0}} \mathbf{u}\left(\mathbf{r}_{0}, t_{0}\right)-\mathbf{u} \cdot \frac{\partial}{\partial t_{0}} \mathscr{G}\right\}\right|_{t_{0}=0} d V_{0} \\
& +\int_{t_{0}=0}^{\infty} \int_{B} \mathscr{G} \cdot \mathbf{f}\left(\mathbf{r}_{0}, t_{0}\right) d V_{0} d t_{0}
\end{aligned}
$$

for $\mathbf{r} \in \mathbf{B}^{\prime}$. If $r \notin B$ the left-hand side of equation (4) is zero. Here, $\mathbf{n}\left(\mathbf{r}_{0}\right)$ and $d S_{0}$ are the outward normal and surface element of $\partial B$ at $\mathbf{r}_{0}, d V_{0}$ is the volume element and $K_{i j k}=C_{i j r s} G_{r k, s}$ is the stress field associated with $\mathscr{G}$.

The second integral in equation (4) contains the influence of initial values and the third gives the effect of body forces acting in $B^{\prime}$. These two integrals are assumed to be known and will henceforth be represented by the vector $\mathbf{F}(r, t)$, which can often be interpreted as an "infinite space" solution to the body force-initial value problem. The first integral contains the effect of the boundary values $\mathbf{u}$ and $\tau$ and is of primary interest here since the boundary values will be regarded as the basic unknown quantities in the problem; if they were known, the displacement at a point $\mathbf{r} \in B^{\prime}$ could be calculated directly from equation (4).

If $\mathbf{r} \rightarrow \partial B$ in equation (4) one obtains an integral equation over $\partial B$ and $t>0$ involving only the boundary values $\mathbf{u}$ and $\tau$ and the known vector $\mathbf{F}$. In doing this there is a difficulty arising from the singularities of $\mathscr{G}$ as $\mathbf{r} \rightarrow \mathbf{r}_{0}$. Before evaluating the contribution of the singularities to the surface integrals it is necessary to become more specific about the choice of $\mathscr{G}$.

Up to this point the only restrictions placed on $\mathscr{G}$ are those provided by equations 
(3), (3a), and (3b), which apply in $B^{\prime}$. These conditions are insufficient to uniquely specify $\mathscr{G}$ in $B$, although equation (4) holds for any tensor satisfying them. In particular, they are insufficient to uniquely specify the singularities of $\mathscr{G}$ as $\mathbf{r} \rightarrow \mathbf{r}_{0}$ for $\mathbf{r}_{0} \in \partial B$ since they do not exclude solutions of equation (3) which contain image sources outside $B$. In the numerical examples to follow the infinite space Green's tensor is used for $\mathscr{G}$, so the BIE derived here will use that tensor. This choice is arbitrary and for some problems it may be advantageous to use other tensors. For example, Das and Aki (1977) use a half-plane Green's tensor and their BIE is of a slightly different form from that obtained here.

The infinite space Green's tensor is (Wheeler and Sternberg, 1968)

$$
\begin{aligned}
4 \pi \rho G_{i j}\left(\mathbf{r}, t ; \mathbf{r}_{0}, 0\right)= & \left\{\frac{3 y_{i} y_{j}}{y^{3}}-\frac{\delta_{i j}}{y}\right\} \int_{1 / \alpha}^{1 / \beta} p \delta(t-p y) d p \\
& +\frac{y_{i} y_{j}}{y^{3}}\left[\frac{1}{\alpha^{2}} \delta(t-y / \alpha)-\frac{1}{\beta^{2}} \delta(t-y / \beta)\right] \\
& +\frac{\delta_{i j}}{y \beta^{2}} \delta(t-y / \beta)
\end{aligned}
$$

where $y_{i}=x_{i_{0}}-x_{i}, y=|\mathbf{y}|$.

The associated stress field is

$$
\begin{aligned}
4 \pi K_{i j k}= & 4 \pi C_{i j r s} G_{r k, s_{0}}= \\
& -6 \beta^{2}\left[\frac{5 y_{j} y_{j} y_{k}}{y^{5}}-\frac{\delta_{i j} y_{k}+\delta_{i k} y_{j}+\delta_{j k} y_{i}}{y^{3}}\right] \int_{1 / \alpha}^{1 / \beta} p \delta(t-p y) d p \\
& +2\left[\frac{6 y_{i} y_{j} y_{k}}{y^{5}}-\frac{\delta_{i j} y_{k}+\delta_{i k} y_{j}+\delta_{j k} y_{i}}{y^{3}}\right]\left[\delta(t-y / \beta)-\left(\frac{\beta}{\alpha}\right)^{2} \delta(t-y / \alpha)\right] \\
& +2 \frac{y_{i} y_{j} y_{k}}{y^{4} \beta}\left[\dot{\delta}(t-y / \beta)-\left(\frac{\beta}{\alpha}\right)^{3} \dot{\delta}(t-y / \alpha)\right] \\
& -\frac{y_{k} \delta_{i j}}{y^{3}}\left[1-2\left(\frac{\beta}{\alpha}\right)^{2}\right]\left[\delta(t-y / \alpha)+\frac{y}{\alpha} \dot{\delta}(t-y / \alpha)\right] \\
& -\frac{\delta_{i k} y_{j}+\delta_{j k} y_{i}}{y^{3}}\left[\delta(t-y / \beta)+\frac{y}{\beta} \dot{\delta}(t-y / \beta)\right] .
\end{aligned}
$$

As $y \rightarrow 0, \mathscr{G}$ and $\mathbf{K}$ become infinite and asymptotically behave like sums of functions of the form $y^{-n}(\partial / \partial t)^{m} \delta(t)$ with $n=2,1,0,-1 \ldots$ and $m=0,1,2 \ldots$

We will evaluate the contributions of the singularities in equation (4) as $\mathbf{r} \rightarrow \mathbf{r}_{1} \epsilon \partial B$ by isolating the singularity and replacing the full integrals over $\partial B$ by their principal values. Let $C\left(\mathbf{r}_{1}, \epsilon\right)$ be a cylinder of radius $\epsilon$ centered on $\mathbf{r}_{1}$ with generator parallel to $\mathbf{n}\left(\mathbf{r}_{1}\right)$ (see Figure $1 \mathrm{a}$ ). The intersection of $\partial B$ with $C\left(\mathbf{r}_{1}, \epsilon\right)$ is a disk about $\mathbf{r}_{1}$ denoted by $S\left(\mathbf{r}_{1}, \epsilon\right)$. The first term of the surface integral in equation (4) may be written as the sum of an integral over $S\left(\mathbf{r}_{1}, \epsilon\right)$ and an integral over the 

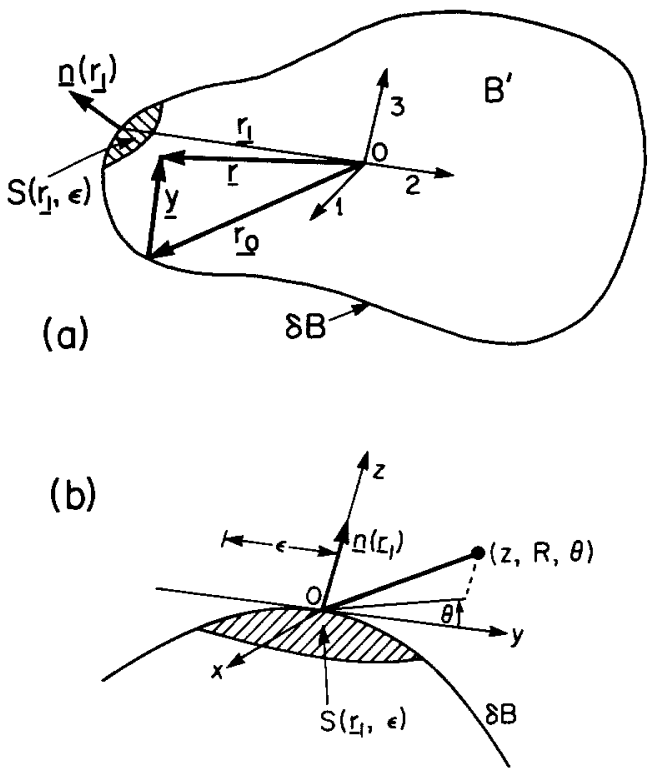

FIG. 1. (a) A homogeneous elastic body $B$ with interior $B^{\prime}$ and boundary $\partial B$. The cap $S\left(\mathbf{r}_{1}, \epsilon\right)$ with normal $n\left(\mathbf{r}_{1}\right)$ contains the singular part of the surfact integral. (b) The cap $S\left(\mathbf{r}_{1}, \epsilon\right)$ and coordinates used in evaluating $S \mathbf{u}\left(\mathbf{r}_{1}, t\right)$ and $S \tau\left(\mathbf{r}_{1}, t\right)$.

remainder of $\partial B$;

$$
\lim _{r \rightarrow r_{1} \in \partial B} \oint_{\partial B} \tau\left(\mathbf{r}_{0}, t_{0}\right) \cdot \mathscr{G}\left(\mathbf{r}, t ; \mathbf{r}_{0}, t_{0}\right) d S_{0}=\lim _{\mathbf{r} \rightarrow r_{1}}\left\{\int_{S\left(\mathbf{r}_{1}, \epsilon\right)} \tau \cdot \mathscr{G} d S_{0}+\int_{\partial B-S\left(\mathbf{r}_{1}, \epsilon\right)} \tau \cdot \mathscr{G} d S_{0}\right\}
$$

and likewise for the displacement integral. As $\epsilon \rightarrow 0$ the second integral on the right becomes a principal value and the first gives the contribution of the singularity. The singular parts of the integrals are then the ordered limits

$$
\begin{gathered}
S \tau\left(\mathbf{r}_{1}, t\right)=\lim _{\epsilon \rightarrow 0} \lim _{\mathbf{r} \rightarrow \mathbf{r}_{1}} \int_{t_{0}=0}^{\infty} \int_{S\left(\mathbf{r}_{1}, \epsilon\right)} \mathscr{G}\left(\mathbf{r}, t ; \mathbf{r}_{0}, t_{0}\right) \cdot \tau\left(\mathbf{r}_{0}, t_{0}\right) d S_{0} d t_{0} \\
S \mathbf{u}\left(\mathbf{r}_{1}, t\right)=\lim _{\epsilon \rightarrow 0} \lim _{r \rightarrow \mathbf{r}_{i}} \int_{t_{0}=0}^{\infty} \int_{S\left(\mathbf{r}_{1}, \boldsymbol{\epsilon}\right)} \mathbf{u}\left(\mathbf{r}_{0}, t_{0}\right) \cdot \mathbf{K}\left(\mathbf{r}, t ; \mathbf{r}_{0}, t_{0}\right) \cdot \mathbf{n}\left(\mathbf{r}_{0}\right) d S_{0} d t_{0} .
\end{gathered}
$$

It is shown in Appendix $A$ that

$$
\begin{aligned}
S \tau\left(\mathbf{r}_{1}, t\right) & =0 \\
S \mathbf{u}\left(\mathbf{r}_{1}, t\right) & =-\frac{1}{2} \mathbf{u}\left(\mathbf{r}_{1}, t\right)
\end{aligned}
$$

if $\partial B$ is sufficiently smooth at $\mathbf{r}_{1}$ and $\mathbf{u}$ and $\tau$ also satisfy smoothness conditions at $\mathbf{r}_{1}$. Hence the BIE is 


$$
\frac{1}{2} \mathbf{u}(\mathbf{r}, t)=\int_{t_{0}=0}^{\infty} P \int_{\partial B}\{\tau \cdot \mathscr{G}-\mathbf{u} \cdot \mathbf{K} \cdot \mathbf{n}\} d S_{0} t_{0}+\mathbf{F}(\mathbf{r}, t)
$$

for $t \geqq 0, \mathbf{r} \in \partial B$, where $\mathscr{G}$ and $\mathbf{K}$ are the infinite space Green's tensor and its associated stress field. It might be noted that this equation is also valid for any tensor whose singularities are the same as those treated on $\partial B$ here and which satisfies equations (3), (3a), and (3b) in the interior $B^{\prime}$.

Two-dimensional problems may be considered as a special case of equation (7) by carrying out the integration on the right side of equation (7) over the translation axis. The two-dimensional BIE is of the same form and in particular the effect of the singularity is exactly the same. Singular kernel contributions of this sort have been considered by Kellogg (1929) for potential theory and by Kupradze (1963) and Mow and Pao (1971) for time-harmonic elastic problems. The effects of the singularity in altering the form of the BIE were the same, since these cases have a similar type of integrable spatial singularity.

In general, one will wish to solve equation (7) for $\mathbf{u}$ or $\tau$, or both subject to some constraint, for $\mathbf{r} \epsilon \partial B$ and $t>0$. For example, if $B$ is to be in contact with another medium the boundary conditions of continuous tractions and displacements across $\partial B$ might be applied and the equations of motion of the contacting medium provide a constraint on $\mathbf{u}$ and $\tau$ in equation (7). For a piecewise homogeneous linear elastic region one may obtain a set of coupled equations like equation (7) over the various boundaries of the medium. The coupling of the equations takes place at common boundaries of the homogeneous subregions. For mixed type boundary value problems in which some components of $\mathbf{u}$ and/or $\tau$ are explicitly given at each point of $\partial B$ equation (7) would be solved for the unknown components at each point.

\section{Numerical APPRoximation OF THE BIE}

We now wish to approximate the BIE (7) for $r \epsilon \partial B, t \in[0, T]$ by a finite system of algebraic equations amenable to solution by digital computers. This will be done by restricting the set of functions admitted as approximate solutions of equation (7) to superpositions from a finite set of functions and by applying equation (7) at only a finite number of points sufficient to yield a solution for the expansion coefficients.

The development in this section will be specialized to the two-dimensional case of antiplane strain which is simpler because only one component each of displacement and traction is nonzero. For this case

$$
\begin{aligned}
u_{1} & =u_{2} \equiv 0 \\
\sigma_{11} & =\sigma_{22}=\sigma_{33}=\sigma_{12} \equiv 0 \\
u_{3} & =u\left(x_{1}, x_{2}, t\right) \\
\tau & =\boldsymbol{\sigma} \cdot \mathbf{n}=\hat{e}_{3} \tau\left(x_{1}, x_{2}, t\right) .
\end{aligned}
$$

Equations (1) and (2) then reduce to

$$
\rho \frac{\partial^{2}}{\partial t^{2}} u=\mu(u, 11+u, 22)+f\left(x_{1}, x_{2}, t\right) .
$$

The infinite space Green's function is 


$$
G\left(\mathbf{r}, t ; \mathbf{r}_{0}, t_{0}\right)=\frac{1}{2 \pi \mu} \frac{H\left(t-t_{0}-y / \beta\right)}{\left[\left(t-t_{0}\right)^{2}-y^{2} / \beta^{2}\right]^{1 / 2}}
$$

$H(t)$ is the Heaviside function, $\beta$ is the shear velocity and

$$
y=\left|\mathbf{r}_{0}-\mathbf{r}\right|=\left[\left(x_{1}-x_{10}\right)^{2}+\left(x_{2}-x_{20}\right)^{2}\right]^{1 / 2} .
$$

The BIE is now on a curve $\partial B$ in the $x_{1}-x_{2}$ plane

$$
\frac{1}{2} u(\mathbf{r}, t)=\int_{t_{0}=0}^{\infty} P \int_{\partial B}\left[G \tau\left(\mathbf{r}_{0}, t_{0}\right)-u \mathbf{K} \cdot \mathbf{n}\right] d S_{0} d t_{0}+F(\mathbf{r}, t) .
$$

Let $\partial B^{*}=\left\{\mathbf{r}_{j}, j=1, \cdots, J\right\} \epsilon \partial B$ be a set of discrete points (nodes) and let $T_{N}{ }^{*}=$ $\left\{t_{n} ; t_{n}=h \Delta t, n=1 \cdots, N\right\}$ be a set of equally spaced times with $t_{N}=T$. The approximations of displacements and tractions on $\partial B$ are, respectively, $u^{*}(\mathbf{r}, t)$ and $\tau^{*}(\mathbf{r}, t)$. A convenient and intuitively useful way of representing $u^{*}$ and $\tau^{*}$ is through interpolation functions

$$
\begin{aligned}
u^{*}(\mathbf{r}, t) & =\sum_{j} \sum_{n} U_{j}^{n}(\mathbf{r}, t) u_{j}^{n} \\
\tau^{*}(\mathbf{r}, t) & =\sum_{j} \sum_{n} T_{j}^{n}(\mathbf{r}, t) \tau_{j}^{n}
\end{aligned}
$$

where $u_{j}^{n}$ and $\tau_{j}^{n}$ are the expansion coefficients. (The indices $j$ and $n$ in this section refer to nodes and times and not to cartesian components as in the previous section.) The expansion coefficients are intended to represent the boundary values through the conditions

$$
\begin{aligned}
& U_{j}^{n}\left(\mathbf{r}_{i}, t_{m}\right)=\delta_{i j} \delta_{m n} \\
& T_{j}^{n}\left(\mathbf{r}_{i}, t_{m}\right)=\delta_{i j} \delta_{m n}
\end{aligned}
$$

for every $\mathbf{r}_{i} \in \partial B^{*}, t_{m} \in T_{N}^{*}$. Then

$$
\begin{aligned}
& u^{*}\left(\mathbf{r}_{i}, t_{m}\right)=u_{i}^{m} \\
& \tau^{*}\left(\mathbf{r}_{i}, t_{m}\right)=\tau_{i}^{m} .
\end{aligned}
$$

Substitution of $u^{*}$ and $\tau^{*}$ for $u$ and $\tau$ in equation (9) for each $\mathbf{r}_{j} \epsilon \partial B^{*}$ and $t_{n} \epsilon T_{N}{ }^{*}$ yields a system of algebraic equations

$$
\begin{aligned}
& u_{j}^{n}=\sum_{m=1}^{N} \sum_{l=1}^{J}\left\{D G_{j i}^{n m} \tau_{i}^{m}+D K_{j i}^{n m} u_{i}^{m}\right\}+2 F\left(\mathbf{r}_{j}, t_{n}\right) \\
& j=1, \cdots, J ; n=1, \cdots, N
\end{aligned}
$$

where

$$
D G_{j i}^{n m}=2 \int_{t_{0}=0}^{\infty} P \int_{\partial B} G\left(\mathbf{r}_{j}, t_{n} ; \mathbf{r}_{0}, t_{0}\right) T_{i}^{m}\left(\mathbf{r}_{0}, t_{0}\right) d S_{0} d t_{0}
$$




$$
D K_{j i}^{n m}=2 \int_{t_{0}=0}^{\infty} P \int_{\partial B} \mathbf{n} \cdot \nabla_{0} G\left(\mathbf{r}_{j}, t_{n} ; \mathbf{r}_{0}, t_{0}\right) U_{i}^{m}\left(\mathbf{r}_{0}, t_{0}\right) d S_{0} d t_{0}
$$

In general there will be $J$ equations (12) and $J$ boundary conditions for each time level $n$. The system of equations (12), will be solved by time stepping, i.e., set $n=1$, solve for the unknown coefficients in (12), then set $n=2, \cdots$. The nature of the algebraic system to be solved at each time step will be determined by the type of boundary condition used and by the interpolation scheme used. In particular, as will be seen, the overall scheme may be made implicit or explicit depending on the type of interpolation used.

At first sight it would appear that there are $(\mathscr{O})\left(N^{2} J^{2}\right)$ discrete kernels $D G_{j i}^{n m}$ and $D K_{j i}^{n m}$ to be calculated. However, if the interpolation functions are assigned time translation properties similar to those of the Green's function, the number of discrete kernels required is $(O)\left(N J^{2}\right)$ in all cases. Suppose that

$$
\begin{aligned}
& U_{i}^{m}(\mathbf{r}, t)=U_{i}^{m+l}(\mathbf{r}, t+l \Delta t) \\
& T_{i}^{m}(\mathbf{r}, t)=T_{i}^{m+l}(\mathbf{r}, t+l \Delta t) .
\end{aligned}
$$

Then from property (3b) of the Green's function one easily obtains

$$
\begin{aligned}
& D G_{j i}^{n m}=D G_{j i}^{n+l m+l} \\
& D K_{j i}^{n m}=D K_{j i}^{n+l m+l}
\end{aligned}
$$

Hereafter the upper (time) indices of $D G_{j i}^{n m}$ will be written unambiguously as $D G_{j i}^{n-m}$ and likewise for $D K_{j i}^{n-m}$. The causality properties of the Green's function also cause some of the discrete kernels to be zero if it is required that

$$
\begin{array}{llll}
U_{j}^{n}(\mathbf{r}, t)=0 & t<t_{n}-p \Delta t & \text { or } & \left|\mathbf{r}-\mathbf{r}_{j}\right|>a \\
T_{j}^{n}(\mathbf{r}, t)=0 & t<t_{n}-q \Delta t & \text { or } & \left|\mathbf{r}-\mathbf{r}_{j}\right|>b
\end{array}
$$

Under these circumstances the interpolator $U_{j}^{n}$ does not transmit information about the motion at $\left(\mathbf{r}_{j}, t_{n}\right)$ to points lying outside a semi-infinite space-time cylinder of radius " $a$ " centered on $\mathbf{r}_{j}$ with generator parallel to the time axis and extending from $t=\infty$ to $t=t_{n}-p \Delta t . T_{j}^{n}$ is similarly causal. Then, since $G\left(\mathbf{r}, t ; \mathbf{r}_{0}, t_{0}\right)=0$ for $\beta\left(t-t_{0}\right)<\left|\mathbf{r}-\mathbf{r}_{0}\right|$ one obtains the analogous relations

$$
\begin{array}{ll}
D K_{j i}^{n-m}=0 & n-m-p<\left(\left|\mathbf{r}_{i}-\mathbf{r}_{j}\right|+a\right) \beta \Delta t \\
D G_{j i}^{n-m}=0 & n-m-q<\left(\left|\mathbf{r}_{i}-\mathbf{r}_{j}\right|+b\right) / \beta \Delta t .
\end{array}
$$

Relations such as equation (17) can thus be used to make the scheme implicit or explicit. For the three-dimensional case a similar result is obtained from the causality condition equation ( $3 a$ ). If an infinite space three-dimensional Green's tensor is used there is an additional relation corresponding to quiescence after passage of the shear wave. 
The properties represented by equations (16) and (18) are discrete analogs of the properties of the analytic Green's function. The spatial translation-rotation symmetries of the free-space Green's function are not present in the discrete versions because the node numbering scheme will not in general correspond to uniform increments of distance and because of the surface orientation involved in the integrals of equations (13) and (14). Exceptions to this exist, for example in uniformly gridded planes and cylinders.

The time translation property, equation (16), makes clear the possibilities for numerical instability. If the type of boundary condition does not change with time, at each time step one must solve a system

$$
\mathscr{A} \cdot \mathbf{C}^{n}=\sum_{m=1}^{n-1} \mathscr{B}^{n-m} \cdot \mathbf{C}^{m}+\mathbf{v}^{n}
$$

where $\mathscr{A}, \mathscr{B}^{1}, \mathscr{B}^{2}, \cdots$ are the same for each time step, $\mathbf{C}^{m}$ is the vector of unknowns at time level $m$ and $\mathbf{v}^{n}$ contains the rest of the terms in equation (12). One might regard this iterative process as similar to a finite difference method on the boundary in which the difference molecule expands backward in time with each step, with the same problems of stability. (This is in fact the approach taken by Burridge, 1969, in solving fracture problems. He discretizes a nonlocal differential operator involving derivatives with respect to time and the tangent to the boundary.) For even the simplest curves $\partial B$ and interpolators the discrete kernels depend in a very complex way on the discretization parameters, and a thorough analysis of stability and consistency is as yet unavailable. Appendix $\mathrm{C}$ contains a brief discussion of these matters for a straight boundary.

The problem of creating an accurate method of solving antiplane elasticity problems is now reduced to the problem of selecting interpolation functions that cause solutions of equation (12) to accurately represent the motion of the body. The specific method presented here was chosen because of its simplicity and intuitive appeal. Its usefulness will be demonstrated in the following section, which deals with a numerical example.

Consider now the situation where $u(\mathbf{r}, t)$ and $\tau(\mathbf{r}, t)$ are known on $\partial B$ up to some time $t_{m}$ and we wish to investigate the motion through the next time step. Let

$$
I_{m}(\mathbf{r}, t)=2 \int_{t_{0}=0}^{t_{m}} P \int_{\partial B}[G \tau-u \mathbf{K} \cdot \mathbf{n}] d S_{0} d t_{0}+2 F(\mathbf{r}, t)
$$

so that $I_{m}\left(\mathbf{r}, t_{m}\right)=u\left(\mathbf{r}, t_{m}\right) . I_{m}(\mathbf{r}, t)$ contains all required information about the motion of $B$ before time $t_{m}$. From equation (12) one obtains

$$
u(\mathbf{r}, t)=2 \int_{t_{0}=t_{m}}^{t+} P \int_{\partial B}[G \tau-u \mathbf{K} \cdot \mathbf{n}] d S_{0} d t_{0}+I_{m}(r, t)
$$

where

$$
t+=\lim _{\epsilon \rightarrow 0+}(t+\epsilon)
$$


If $t$ is restricted to the interval $\left[t_{m+1}, t_{m}\right]$ and if $\beta \Delta t$ is small compared to the dimensions of $\partial B$ the integration over $\partial B$ in equation (19) will be over a relatively small region about $\mathbf{r}$, since $G$ and $\mathbf{K}$ are nonzero only within the backward causality cone of $(\mathbf{r}, t)$. (See Appendix B, Figures 5 and 6.) In this sense the point $(\mathbf{r}, t)$ is isolated because it receives information on the motion of $\partial B$ after $t_{m}$ only from a small region.

Accordingly, the interpolation functions should not destroy this isolation and in general should permit accurate modeling of the flow of information through the various causality cones of the node points. Over short times and distances, i.e., near the apex of the backward causality cone where the Green's function is most strongly singular, the nature of the interaction of the nodes is strongly influenced by the type of interpolation used. Therefore, some care should be taken in the selection of interpolators so that they accurately represent short scale dynamics and do not transmit information noncausally to nearby nodes. The causality requirements indicate that the interpolation functions should be highly localized in time and space.

In order to calculate the values of the discretized kernels $D G_{i j}^{n}$ and $D K_{i j}^{n}$ it is necessary to introduce a parameterization of $\partial B$. (This calculation must be carried out from analytic formulas near the singularities of $G$ and $\mathbf{K}$.) There are many ways of approximating $\partial B$ by fitting a curve through the points of $\partial B^{*}$, but the simplest one will be used here. Let $D B$ be an approximation of $\partial B$ consisting of a series of connected straight line segments $A_{j}$ of length $L_{j}$ through the points $\mathbf{r}_{j}$ of $\partial B^{*}$ and tangent to $\partial B$ at $\mathbf{r}_{j}$ (see Figure 2). If $\beta \Delta t<\min _{i=1, \cdots, J}\left(L_{i} / 2\right)$ and $\mathbf{r}=\mathbf{r}_{j} \epsilon \partial B^{*}$ in equation (19) the spatial projection of the portion of the backward causality cone of $\left(\mathbf{r}_{j}, t\right)$ with $t_{m+1} \geqq t \geqq t_{m}$ lies entirely within $A_{j}$. Substituting $u^{*}$ and $\tau^{*}$ in equation (19) one obtains

$$
\begin{array}{r}
u^{*}\left(\mathbf{r}_{j}, t\right)=\int_{t_{m}}^{t+} \int_{A_{j}} 2 G\left(\mathbf{r}_{j}, t ; \mathbf{r}_{0}, t_{0}\right) \tau^{*}\left(\mathbf{r}_{0}, t_{0}\right) d S_{0} d t_{0}+I_{m}{ }^{*}\left(\mathbf{r}_{j}, t\right), \\
j=1, \cdots, J
\end{array}
$$

since $K\left(\mathbf{r}_{j}, t ; \mathbf{r}_{0}, t\right) \cdot \mathbf{n}\left(\mathbf{r}_{0}\right)=0$ for $\mathbf{r}_{0} \in A_{j} . I_{m}{ }^{*}$ is the approximation to $I_{m}$ obtained by using $u^{*}$ and $\tau^{*}$ in the definition of $I_{m}$. Upon setting $t=t_{m+1}$ in equation (20) one obtains equation (12) simplified by using the approximation $D B$ of $\partial B$.

The objective here is to obtain a general purpose linear relation among the coefficients $u_{j}^{m}, \tau_{j}^{m}$ that accurately represents the physical behavior of the elastic body. In the analytic BIE the value of $u(\mathbf{r}, t)$ depends only on the boundary values before time $t$. Thus it is physically acceptable that upon setting $t=t_{m+1}$ in equation (20) it contains only $u_{j}^{m+1}, u_{j}^{m}, \cdots, u_{j}{ }^{1}(j=1, \cdots, J)$. Furthermore in view of the identification

$$
I_{m}\left(\mathbf{r}_{j}, t_{m}\right)=u\left(\mathbf{r}_{j}, t_{m}\right)
$$

it is appropriate that $I_{m}{ }^{*}$ depend only on $u_{j}^{m}, u_{j}^{m-1}, \cdots, u_{j}^{1}(j=1, \cdots, J)$. Similar arguments apply for $\tau^{*}$, therefore let $p=q=1$ in equation (17). With this restriction, at time step $(m+1)$ the algebraic equation (12) will be solved for the unknowns among $u_{j}^{m+1}, \tau_{j}^{m+1},(j=1, \cdots, J)$, since these are the highest time level coefficients appearing there. 
If $\tau^{*}\left(\mathbf{r}_{0}, t_{0}\right), \mathbf{r}_{0} \in A_{j}, t_{0} \in\left[t_{m+1}, t_{m}\right]$ depends on coefficients of higher time level than $m$ only through $\tau_{j}{ }^{m+1}$, then since $I_{m}{ }^{*}$ depends only on $\tau_{i}{ }^{m}, u_{i}{ }^{m}, \tau_{i}{ }^{m-1}, u_{i}{ }^{m-1}, \cdots$, $i=1, \cdots, J$ it is apparent that equation (20) will contain only $\tau_{j}^{m+1}, u_{j}^{m+1}$ from time level $(m+1)$ for a particular value of $j$. That is, nodes do not communicate over time intervals less than $\Delta t$ because $2 \beta \Delta t / L_{i} \leqq 1$, and because the Green's function is causal and the interpolation functions are spatially localized. This causes the iterative scheme to be explicit and is a direct result of preserving the causal nature

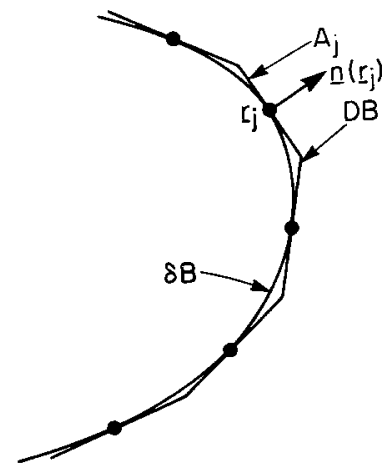

FIG. 2. Approximation of a boundary $\partial B$ by its discretization $D B$.

of the analytic Green's function in constructing the discrete kernels. The simplest type of spatial interpolation that has this localization property is piecewise constant

$$
\begin{aligned}
T_{j}^{n}(\mathbf{r}, t) & = \begin{cases}T_{j}^{n}(t) & \mathbf{r} \in A_{j}, t \geqq t_{n}-\Delta t \\
0 & \text { otherwise }\end{cases} \\
U_{j}^{n}(\mathbf{r}, t) & = \begin{cases}U_{j}^{n}(t) & \mathbf{r} \in A_{j}, t \geqq t_{n}-\Delta t \\
0 & \text { otherwise. }\end{cases}
\end{aligned}
$$

The use of piecewise constant interpolation for displacements is not necessary for the purpose of constructing an explicit scheme, but it makes it relatively easy to calculate the kernels $D K_{i j}^{n}$. The use of interpolators with separable space and time dependence is in itself noncausal, since it allows transmission of signals from a node $\mathbf{r}_{j}$ to the extremity of the line element $A_{j}$ instantaneously. With the use of very localized interpolators the effect of errors introduced by this is not expected to be large unless wavelengths comparable to the node separation are encountered.

It now remains only to choose the time dependence of the interpolators $U_{j}^{n}(t)$, $T_{j}^{n}(t)$. Since

$$
\tau^{*}(\mathbf{r}, t)=\tau^{*}\left(\mathbf{r}_{j}, t\right), \mathbf{r} \in A_{j}
$$

the spatial integration in equation (20) may be carried out to give

$$
u^{*}\left(\mathbf{r}_{j}, t\right)=\frac{\beta}{\mu} \int_{t_{m}}^{t} \tau^{*}\left(\mathbf{r}_{j}, t_{0}\right) d t_{0}+I_{m}^{*}\left(\mathbf{r}_{j}, t\right)
$$


The time dependence of $I_{m}{ }^{*}$ will in general be unknown, so the best that can be done to ensure suitability of the time interpolation is to require consistency of $U_{j}^{n}(t)$ with $T_{j}^{n}(t)$. In order to keep them localized in time let

$$
T_{j}^{n}(t)= \begin{cases}1 & t_{n} \geqq t \geqq t_{n-1} \\ 0 & \text { otherwise. }\end{cases}
$$

Since the displacement in equation (22) is related to a time integral of the tractions a suitable type of interpolation for displacements is piecewise linear

$$
U_{j}^{n}(t)= \begin{cases}1- & \left|t-t_{n}\right| / \Delta t, \quad t_{n+1} \geqq t \geqq t_{n-1} \\ 0 & \text { otherwise. }\end{cases}
$$

This defines the interpolation scheme used in the numerical example. (It will be denoted as $D_{0}$ in Appendix C.)

The use of piecewise constant time interpolation for tractions is not altogether arbitrary. If piecewise linear interpolation is used, as for displacements, it is found that the iterative scheme is unstable when solving for tractions. An explanation of this effect is given in Appendix C.

\section{A Numerical Example}

In this section we consider an elementary problem in elastic wave propagation. It is the problem of finding the $S H$ motion of two welded half-planes under the influence of a two-dimensional point source. The point source is located in the medium with lower velocity, giving rise to a head wave. The geometry of the problem is shown in Figure 3. The interface extends to infinity in both directions. The numerical grid consists of 48 line segments of length $L$. Note that it is truncated at nodes 1 and 48 (see Figure 3). The source is located above nodes 24 and 25 . The time step $\Delta t$ was selected to be the largest time step for an explicit scheme

$$
Q_{2}=\frac{\beta_{2} \Delta t}{L}=\frac{1}{2}
$$

(numerical subscripts refer here to medium 1 or medium 2).

The time dependence of the point source was a quadratic: $f(\mathbf{r}, t)=\delta\left(\mathbf{r}-\mathbf{r}_{s}\right) h(t)$ where

$$
h(t)=\left\{\begin{array}{l}
0 ; \quad t<0, t>2 \tau_{s} \\
\frac{t\left(\tau_{s}-t\right)}{\tau_{s}^{2}} ; 0<t<\tau_{s} \\
\frac{\left(t-\tau_{s}\right)\left(2 \tau_{s}-t\right)}{\tau_{s}^{2}} ; \quad \tau<t<2 \tau_{s} .
\end{array}\right.
$$

For the example presented, $\tau_{s}$ was chosen to be $10 \Delta t$, so the free space wavelength of the disturbance is about 5 grid lengths in medium 1. 
The free space wave form, $F(\mathbf{r}, t)$ was calculated using

$$
F(\mathbf{r}, t)=\int_{0}^{t} G_{1}\left(\mathbf{r}, t ; \mathbf{r}_{s}, t_{0}\right) h\left(t_{0}\right) d t_{0} .
$$

For this geometry the discrete kernel has the symmetry properties

$$
D G_{i+l, j+l}^{m}=D G_{i j}^{m}=D G_{j i}^{m}
$$

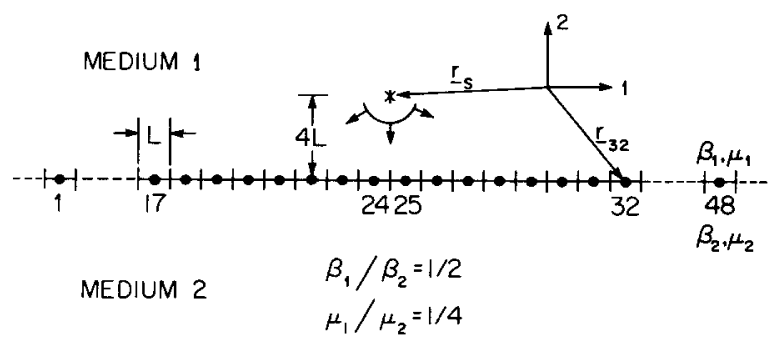

Fig. 3. Geometry of the numerical example. It consists of two half-planes with infinite welded contact. Also shown is the discretization of the boundary, truncated at nodes 1 and 48 . The point source is at $\mathbf{r}_{s}$.

Let $k=|i-j|$. The kernel for the interpolation scheme given by equations (21), (23), and (24) is then

$$
D G_{i j}^{m}=\frac{Q L}{\pi \mu}\left(E_{m}^{+1}-1\right)\left(E_{k}^{+1 / 2}-E_{k}^{-1 / 2}\right) m H(m) g\left(\frac{k}{m Q}\right)
$$

where $Q=\beta \Delta t / L . E_{k}^{b}$ is the translation operator; $E_{k}^{b} f(k, m)=f(k+b, m)$, and

$$
g(\eta)=\left\{\begin{array}{l}
\eta \cosh ^{-1}\left(|\eta|^{-1}\right)+\sin ^{-1}(\eta),|\eta| \leqq 1 \\
\frac{\pi}{2} \operatorname{sgn}(\eta),|\eta| \geqq 1
\end{array}\right.
$$

The discretized BIE for this problem is

$$
\begin{gathered}
u_{l j}^{n}=\sum_{i=1}^{48} \sum_{m=1}^{n} D G_{1 i j}^{n-m} \tau_{l i}^{m}+2 F\left(r_{j}, t_{n}\right) \\
u_{2 j}^{n}=\sum_{i=1}^{48} \sum_{m=1}^{n} D G_{2 i j}^{n-m} \tau_{2 i \cdot}^{m}
\end{gathered}
$$

The displacements do not appear on the right-hand side because $D K_{i j}^{m}=0$, for this geometry. Note that truncation of the grid is equivalent to making the boundary traction-free outside the grid in this particular problem. By application of the boundary conditions

$$
\begin{aligned}
u_{1 j}^{n} & =u_{2 j}^{n} \\
-\tau_{1 j}^{n} & =\tau_{2 j}^{n}
\end{aligned}
$$


equations (27) and (28) were solved by time stepping. The analytic solution was obtained using integral transform techniques and the Cagniard-de Hoop transform inversion method, which gives the exact solution in closed form for this problem.

The numerical solution is shown for three nodes along with the exact solution in Figure 4. As can be seen, the results are quite good for the entire time series. The maximum error

$$
\epsilon_{j}=\max _{n}\left|u_{j}^{n}-u_{e x}\left(\mathbf{r}_{j}, t_{n}\right) / \max _{n}\right| u_{e x}\left(\mathbf{r}_{j}, t_{n}\right) \mid
$$

is about 5 per cent and occurs at the onset of the direct wave at nodes remote from the source. (See Figure 4, node 1, time steps 96 and 116.) The error at other times is smaller ( $<2$ per cent) and all errors decrease approximately linearly with $L / \beta_{1} \tau_{s}$ for fixed $Q_{2}$ and $\beta_{1} / \beta_{2}$. The anomalously large onset error is eliminated when a time function with smoother onset is used. A rather interesting result is that the numerical solution is also good at node 1 , which is located at the truncation of the BIE grid.

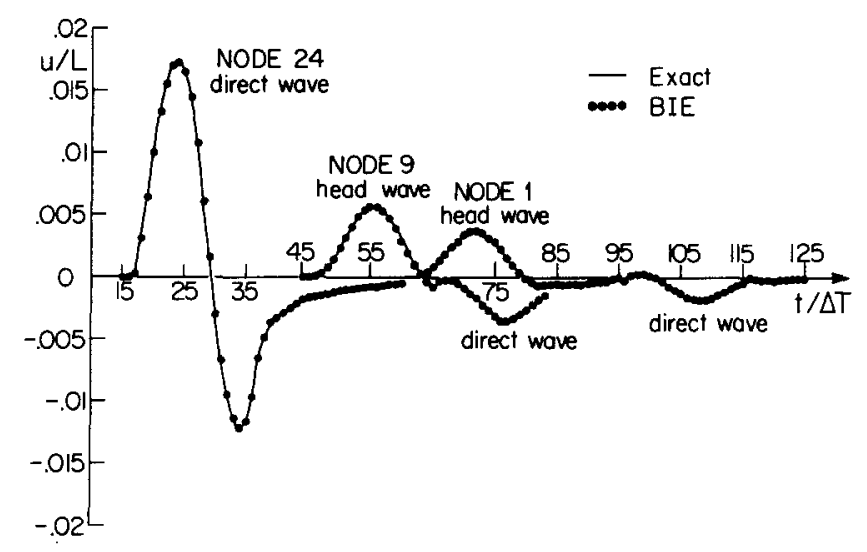

FIG. 4. Analytic and numerical solutions for nodes 24,9 , and 1 .

This can be understood through an examination of the analytic solution to the stress field near the boundary far from the source. It is found that the traction on the interface is sufficiently small near node 1 so that the approximation of zero tractions outside of the numerical grid does not cause the quadratures in equations (27) and (28) to be greatly inaccurate. It should also be noted that the head wave, which is well developed at nodes 9 and 1, is not distorted in the numerical solution. This suggests that numerical dispersion is not a significant effect along straight boundaries in the BIE method.

\section{CONCLUSION}

The boundary integral equation formulation of elastodynamics provides a useful foundation for the creation of a numerical procedure for the solution of boundary value problems. Since the problem is directly posed in terms of the boundary values of displacements and stresses, the unknown boundary values may be found without calculating the motion of interior points. This reduces the spatial dimensionality of the problem by one and may eliminate some problems found in volume gridding methods, such as numerical dispersion. The dynamic behavior of the medium is represented entirely by the Green's function, so it is necessary that the discretization of the analytic BIE be consistent with the physical nature of the Green's function. 
In particular, the interpolation scheme must not transmit information noncausally and must accurately represent the short-scale dynamics of the medium. In the numerical procedure used here, this was accomplished by using a very localized interpolation scheme. The performance of the method was found to be very adequate in a simple numerical problem.

\section{ACKNOWLEDGMENT}

The study presented here was carried out with the support of the National Science Foundation, Earth Sciences Division under Contract EAR76-22624.

\section{APPENDIX A}

We wish to evaluate the limits

$$
\begin{gathered}
S \tau\left(\mathbf{r}_{1}, t\right)=\lim _{\epsilon \rightarrow 0} \lim _{\mathbf{r} \rightarrow \mathbf{r}_{1}} \int_{t_{0}=0}^{\infty} \int_{S\left(\mathbf{r}_{1}, \epsilon\right)} \mathscr{G}\left(\mathbf{r}, t ; \mathbf{r}_{0}, t_{0}\right) \cdot \tau\left(\mathbf{r}_{0}, t_{0}\right) d S_{0} d t_{0} \\
S \mathbf{u}\left(\mathbf{r}_{1}, t\right)=\lim _{\epsilon \rightarrow 0} \lim _{\mathbf{r} \rightarrow \mathbf{r}_{1}} \int_{t_{0}=0}^{\infty} \int_{S\left(\mathbf{r}_{1}, \epsilon\right)} \mathbf{u}\left(\mathbf{r}_{0}, t_{0}\right) \cdot \mathbf{K}\left(\mathbf{r}, t ; \mathbf{r}_{0}, t_{0}\right) \cdot \mathbf{n}\left(\mathbf{r}_{0}\right) d S_{0} d t_{0} .
\end{gathered}
$$

If $\mathbf{r}_{1}$ is located on a portion of $\partial B$ which satisfies the Liapunov smoothness conditions locally there is a continuously differentiable function $h(R, \theta)$ and constants $A$ and $\delta$ such that

$$
z=h(R, \theta) ;|h(R, \theta)|<A R^{1+\delta}, 0<\delta \leqq 1
$$

is a description of $\partial B$ about $\mathbf{r}_{1}$. The coordinates $(z, R, \theta)$ refer to a cylindrical coordinate system centered on $\mathbf{r}_{1}$ with the $z$-axis parallel to $\mathbf{n}\left(\mathbf{r}_{1}\right)$ (see Figure $1 b$ ).

It will be assumed that $\mathbf{u}(\mathbf{r}, t)$ and $\tau(\mathbf{r}, t)$ satisfy Hölder conditions as a function of space about $(\mathbf{r}, t)$ i.e.

$$
\left|\tau_{i}\left(\mathbf{r}_{1}, t\right)-\tau_{i}(\mathbf{r}, t)\right|<C\left|\mathbf{r}-\mathbf{r}_{1}\right|^{\gamma}, 0<\gamma \leqq 1
$$

and likewise for $\mathbf{u}\left(\mathbf{r}_{1}, t\right)$. $C$ is a constant. It will also be assumed that $\tau$ and $\partial / \partial t \mathbf{u}$ satisfy Hölder conditions near $\left(\mathbf{r}_{1}, t\right)$ as a function of time.

To show that $S \tau=0$ it is only necessary to demonstrate that a representative term of $S \tau$ is zero, e.g., $S \tau_{i}{ }^{*}=0$ where

$$
S \tau_{i}^{*}=\lim _{\epsilon \rightarrow 0} \lim _{\mathrm{r} \rightarrow \mathrm{r}_{1}} \int_{t_{0}=0}^{\infty} \int_{S\left(\mathrm{r}_{1}, \epsilon\right)} \tau_{i} \frac{\delta\left(t-t_{0}-y / \alpha\right)}{y} d S_{0} d t_{0}
$$

The other type of term of $\mathscr{G}$ has the same order of singularity, since $\left|y_{i} y_{j} / \mathrm{y}^{2}\right| \leqq 1$, and its integral in the limit must also be zero.

If the order of integration of (A.5) is reversed and the integration over time is carried out one obtains

$$
S \tau_{i}^{*}\left(\mathbf{r}_{1}, t\right)=\lim _{\epsilon \rightarrow 0} \lim _{\mathbf{r} \rightarrow \mathbf{r}_{1}} \int_{S\left(\mathbf{r}_{1}, \epsilon\right)} \frac{\tau_{i}\left(\mathbf{r}_{0}, t-y / \alpha\right)}{y} d S_{0} .
$$


Since $\tau$ satisfies a Hölder condition as a function of time an expression similar to (A.4) holds

$$
\tau_{i}\left(\mathbf{r}_{0}, t-y / \alpha\right)=\tau_{i}\left(\mathbf{r}_{0}, t\right)+\mathscr{O}\left(y^{\eta}\right)
$$

where

$$
0<\eta \leqq 1
$$

Thus an expansion of $\tau_{i}$ about $\left(\mathbf{r}_{1}, t\right)$ is possible which is of the form

$$
\tau_{i}\left(\mathbf{r}_{0}, t-y / \alpha\right)=\tau_{i}\left(\mathbf{r}_{1}, t\right)+\mathscr{O}\left(y^{\eta}\right)+\mathscr{O}\left(y^{\gamma}\right)
$$

as $\mathbf{r} \rightarrow \mathbf{r}_{1}$. Since $y^{\gamma-1}<y^{-1}$ as $y \rightarrow 0$ it is sufficient to show that

$$
\lim _{\epsilon \rightarrow 0} \lim _{\mathbf{r} \rightarrow \mathbf{r}_{1}} \int_{s\left(\mathbf{r}_{1}, \epsilon\right)} \frac{1}{y} d S_{0}=0 .
$$

The vanishing of less singular contributions then follows.

In the local coordinate system, if $\mathbf{r} \rightarrow \mathbf{r}_{1}$ along the $z$-axis

$$
y^{2}=R_{0}^{2}+\left(\left|\mathbf{r}-\mathbf{r}_{1}\right|-h\left(R_{0}, \theta\right)\right)^{2}
$$

using (A.3) for small $R_{0}$

$$
\frac{1}{y}=\frac{1}{\left[{\left.R_{0}{ }^{2}+{z^{\prime}}^{2}\right]^{1 / 2}}_{1}\right.}\left[1+\mathscr{O}\left(\frac{A z^{\prime} R_{0}{ }^{1+\delta}}{{R_{0}}^{2}+{z^{\prime}}^{2}}\right)\right]
$$

where $z^{\prime}=\left|\mathbf{r}-\mathbf{r}_{1}\right|$. Also

$$
d S_{0}=\left[1+\mathscr{O}\left(R_{0}^{\delta}\right)\right] R_{0} d R_{0} d \theta
$$

Neglecting the higher order, less singular terms, the integral in (A.7) becomes

$$
\lim _{\epsilon \rightarrow 0} \lim _{z^{\prime} \rightarrow 0} 2 \pi \int_{R_{0}=0}^{\epsilon} \frac{R_{o} d R_{o}}{\left[R_{0}^{2}+z^{\prime 2}\right]^{1 / 2}}=2 \pi \lim _{\leftrightarrow \rightarrow 0} \lim _{z^{\prime} \rightarrow 0}\left[\left(\epsilon^{2}+z^{\prime 2}\right)^{1 / 2}-z^{\prime}\right]=0
$$

This proves (A.7) and therefore $S \tau^{*}\left(\mathbf{r}_{1}, t\right)=0$ and $S \tau\left(\mathbf{r}_{1}, t\right)=0$. The proof of (A.7) is easily extended to show that

$$
\lim _{\epsilon \rightarrow 0} \lim _{\mathbf{r} \rightarrow \mathbf{r}_{1}} \int_{S\left(\mathbf{r}_{1}, \epsilon\right)} \frac{1}{y^{2-\xi}} d S_{0}=0 \quad \text { for } \xi>0
$$

In evaluating $S \mathbf{u}\left(\mathbf{r}_{1}, t\right)$ we note that the only possible nonzero contributions will 
come from terms of $\mathrm{K}$ of the form

$$
\delta\left(t-t_{0}-y / \alpha\right) \frac{y_{i} y_{j} y_{k}}{y^{5}} \text { and } \delta\left(t-t_{0}-y / \alpha\right) \frac{y_{i} \delta_{j k}}{y^{3}}
$$

since all other terms are of order $1 / y$ as $y \rightarrow 0$ and $\partial \mathbf{u} / \partial t$ satisfies a Hölder condition. The dot product of $\mathbf{n}\left(\mathbf{r}_{0}\right)$ with terms of this form gives three types of singular terms

$$
\frac{y_{i} y_{j} y_{k} n_{k}}{y^{5}}, \frac{\delta_{i j} y_{k} n_{k}}{y^{3}}, \text { and } \frac{y_{i} n_{k}}{y^{3}}
$$

A property of the Liapunov surface is that for $\mathbf{r}_{1}, \mathbf{r}_{0} \epsilon \partial B$

$$
\left|\mathbf{n}\left(\mathbf{r}_{1}\right) \cdot\left(\mathbf{r}_{1}-\mathbf{r}_{0}\right)\right|<A\left|\mathbf{r}_{1}-\mathbf{r}_{0}\right|^{1+\delta}
$$

so the first two terms of (A.8) are of order $1 / y^{2-\delta}$ as $\mathbf{r} \rightarrow \mathbf{r}_{1}$ and by the previous arguments give no contribution to the integral of (A.2) in the limit. The only remaining singular integrals are then of the form

$$
\lim _{\epsilon \rightarrow 0} \lim _{\mathbf{r} \rightarrow \mathbf{r}_{1}} \int_{S\left(\mathbf{r}_{1}, \boldsymbol{\epsilon}\right)} \frac{u_{i}\left(\mathbf{r}_{0}, t-y / \alpha\right)}{y^{3}} y_{i} \dot{n}_{k} d \delta_{o}
$$

First, the function $\mathbf{u}\left(\mathbf{r}_{0}, t-y / \alpha\right)$ may be expanded about $\left(\mathbf{r}_{1}, t\right)$ and the higher order terms discarded as before. The normal $\mathbf{n}\left(\mathbf{r}_{0}\right)$ also varies smoothly about $\mathbf{r}_{1}$

$$
\mathbf{n}\left(\mathbf{r}_{0}\right)=\mathbf{n}\left(\mathbf{r}_{1}\right)+\mathscr{O}\left(\left|\mathbf{r}_{1}-r_{0}\right|^{\delta}\right)
$$

Thus (A.9) is equivalent to

$$
u_{i}\left(\mathbf{r}_{1}, t\right) \lim _{\epsilon \rightarrow 0} \lim _{r \rightarrow r_{1}} \int_{S\left(\mathbf{r}_{1}, \epsilon\right)} \frac{y_{i} n_{k}\left(\mathbf{r}_{1}\right)}{y^{3}} d S_{0} .
$$

It is easily seen from symmetry arguments that the tangential components of $y$, when integrated on a circle about $\mathbf{r}_{1}$ in (A.10), will give no contribution to the integral, so (A.10) is equivalent to

$$
u_{i}\left(\mathbf{r}_{1}, t\right) \delta_{i k} \lim _{\epsilon \rightarrow 0} \lim _{\mathbf{r} \rightarrow \mathbf{r}_{1}} \int_{S\left(\mathbf{r}_{1}, \epsilon\right)} \frac{\mathbf{z}_{o}-\mathbf{z}}{y^{3}} d S_{0} .
$$

By use of the expressions for $y$ and $d S_{o}$ in the local coordinate system one obtains from (A.11) the equivalent integral

$$
u_{k}\left(\mathbf{r}_{1}, t\right) \lim _{\epsilon \in 0} \lim _{z^{\prime} \rightarrow 0} \int \frac{-2 \pi z^{\prime}}{\left[R_{o}{ }^{2}+{z^{\prime}}^{2}\right]^{3 / 2}} R_{o} d R_{o}
$$


where higher order terms have been discarded. This is easily shown to equal $-2 \pi u_{k}\left(\mathbf{r}_{1}, t\right)$. If the various terms of the form (A.9) are summed one obtains the result

$$
S \mathbf{u}\left(\mathbf{r}_{1}, t\right)=-\frac{1}{2} \mathbf{u}\left(\mathbf{r}_{1}, t\right)
$$

\section{APPENDIX B}

The characteristic surface of equation (18) with apex $\left(\mathrm{r}_{1}, t_{1}\right)$ is the conical space time surface (Figure 6) described by

$$
t-t_{1}=\left|\mathbf{r}-\mathbf{r}_{1}\right| / \beta \text {. }
$$

This is the surface along which wave fronts with discontinuous derivatives originating at $\left(\mathbf{r}_{1}, t_{1}\right)$ may propogate outward. This will be called the forward characteristic

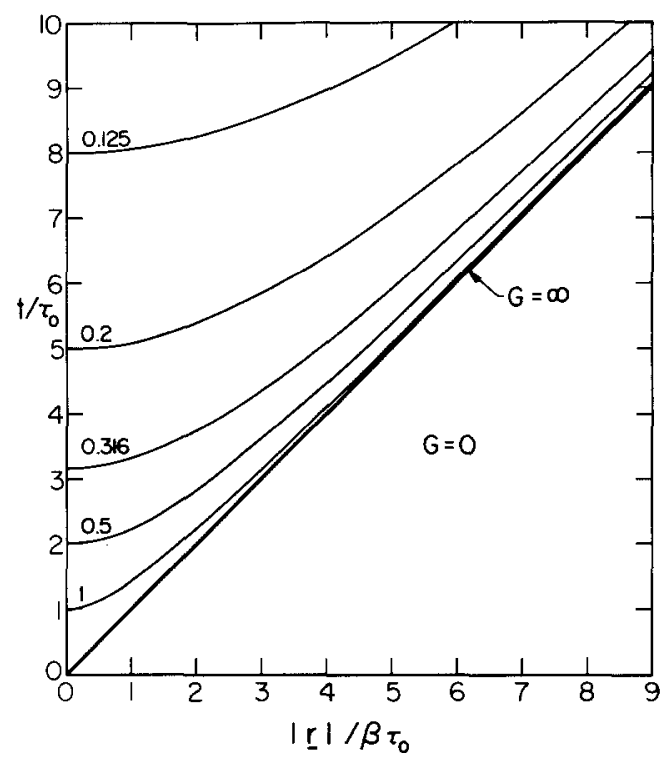

FIG. 5. Contours of the two-dimensional $S H$ Green's function in space and time. $\tau_{0}$ is an arbitrary scaling constant with units of time.

surface to distinguish it from the surface

$$
t-t_{1}=-\left|\mathbf{r}-\mathbf{r}_{1}\right| / \beta
$$

which will be called the backward characteristic surface of $\left(\mathbf{r}_{1}, t_{1}\right)$. Discontinuous wave fronts received at $\left(\mathbf{r}_{1}, t_{1}\right)$ must originate on this surface. The region

$$
t-t_{1} \leqq-\left|\mathbf{r}-\mathbf{r}_{1}\right| / \beta
$$

is the forward causality cone of $\left(\mathbf{r}_{1}, t_{1}\right)$ and is the region into which smooth signals may propogate from $\left(\mathbf{r}_{1}, t_{1}\right)$. The corresponding backward causality cone of $\left(\mathbf{r}_{1}, t_{1}\right)$ 
is the region

$$
t-t_{1} \leqq-\left|\mathbf{r}-\mathbf{r}_{1}\right| / \beta
$$

This is the region from which smooth signals may be received at $\left(\mathbf{r}_{1}, t_{1}\right)$. Courant and Hilbert (1966) have a discussion of characteristics in several space dimensions.
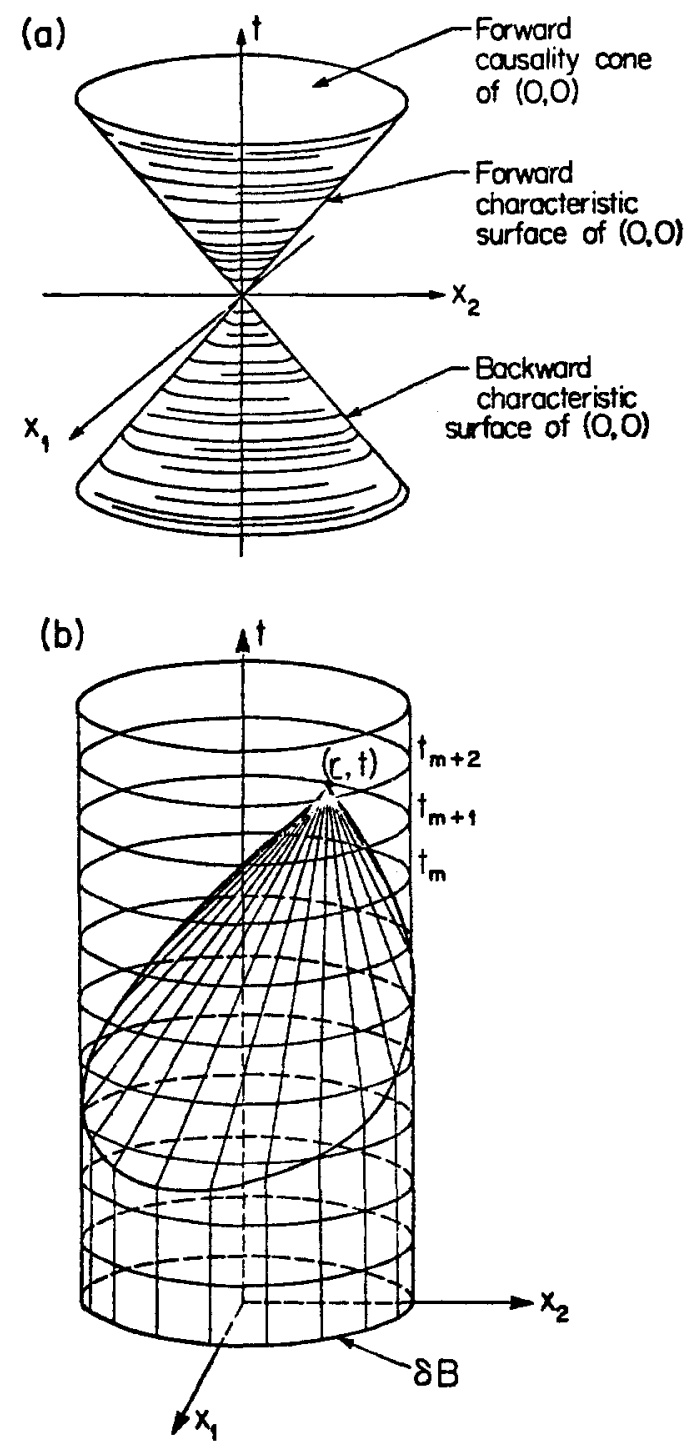

FIG. 6. (a) The causality cones for the two-dimensional scalar wave equation. (b) A depiction of the space time intersection of the causality cone of a point on the boundary of a circle with the circle.

The Green's function $G$ may be written as

$$
G=\frac{1}{2 \pi \mu} \frac{H\left(t-t_{0}-\left|\mathbf{r}-\mathbf{r}_{0}\right| / \beta\right)}{\left[t-t_{0}-\left|\mathbf{r}-\mathbf{r}_{0}\right| / \beta\right]^{1 / 2} \cdot\left[t-t_{0}+\left|\mathbf{r}-\mathbf{r}_{0}\right| / \beta\right]^{1 / 2}} .
$$

This is the displacement due to a point force $\delta\left(\mathbf{r}-\mathbf{r}_{0}\right) \delta\left(t-t_{0}\right)$. Accordingly, it is singular along the forward characteristic cone of $\left(\mathbf{r}_{1}, t_{0}\right)$ and smooth in the interior of the forward causality cone of $\left(\mathbf{r}_{0}, t_{0}\right)$ (see Figure 5 ). The singularity is weaker at 
larger distances. When this is used in equation (9) it is apparent that the point $(\mathbf{r}, t)$ receives discontinuous signals only from points $\left(\mathbf{r}_{0}, t_{0}\right)$ in the backward characteristic surface of $(\mathbf{r}, t)$ and smooth signals from the interior of the backward causality cone. Note that the integration in equation (9) must include the backward characteristic surface.

For three-dimensional and plane strain problems both compressional and shear waves are present and there are correspondingly more characteristic surfaces upon which discontinuous wave fronts may propogate.

\section{APPENDIX C}

The $a$ priori estimation of errors involved in the numerical technique presented here is of considerable practical interest. The method is different from the familiar volume gridding methods in that the interative operator is nonlocal, its elements do not depend on the discretization parameters in a simple way and the process of numerical differentiation is substantially absent. Lacking clear analogies to other well-studied methods, it is important to see what effects the various approximations have in general. Since the general error estimates will involve some unknown coefficients it is equally important to compare numerical and exact solutions to a number of representative problems. This will be carried out in another paper which will also deal with other practical matters.

The analytic antiplane strain BIE, equation (9), may be written in the operational form

$$
u(\mathbf{r}, t)=L G(\partial B ; \mathbf{r}, t) \cdot \tau-L K(\partial B ; \mathbf{r}, t) \cdot u+2 F(\mathbf{r}, t)
$$

The linear functionals $L G$ and $L K$ are defined in equation (9) and depend on $\partial B$ and the particular Green's function in use. When this equation is discretized the displacement $u$ and traction $\tau$ are replaced by their approximations $u^{*}$ and $\tau^{*}$. Also, since the inhomogeneous term $F$ must be represented discretely in any numerical calculations it is approximated in some manner by $F^{*}$. The boundary $\partial B$ may be approximated to any desired accuracy by $D B$ while the number of node points is held constant. (A simple redefinition of the interpolaters is required.) The discrete kernels may be calculated to an accuracy limited only by the word size and the accuracy of any library functions in use. Since the relevant calculations need only be carried out once for a given elastic body and interpolation scheme, it is useful to assume that the discrete kernels are calculated exactly. In any case, near the singularity they must be calculated from analytic formulas, which will presumably be of very high accuracy. Under these circumstances the discretized BIE is the algebraic version of

$$
\begin{array}{r}
u^{*}\left(\mathbf{r}_{j}, t_{n}\right)=L G\left(\partial B ; \mathbf{r}_{j}, t_{n}\right) \cdot \tau^{*}-L K\left(\partial B ; \mathbf{r}_{j}, t_{n}\right) \cdot u^{*}+2 F^{*}\left(\mathbf{r}_{j}, t_{n}\right) \\
j=1, \ldots, J ; n=1, \ldots, N
\end{array}
$$

The order of accuracy of this equation is then the order of quantity

$$
\epsilon_{j}^{n}=L G\left(\partial B ; \mathbf{r}_{j}, t_{n}\right) \cdot\left(\tau-\tau^{*}\right)-L K \cdot\left(u-u^{*}\right)+2\left(F-F^{*}\right)
$$


where $u, \tau$ and $F$ satisfy (C.1) exactly and

$$
\begin{gathered}
u_{j}^{n}=u^{*}\left(\mathbf{r}_{j}, t_{n}\right)=u\left(\mathbf{r}_{j}, t_{n}\right) \\
\tau_{j}^{n}=\tau^{*}\left(\mathbf{r}_{j}, t_{n}-\Delta t / 2\right)=\tau\left(\mathbf{r}_{j}, t_{n}-\Delta t / 2\right) .
\end{gathered}
$$

The order of $\epsilon_{j}^{n}$ is easily found as follows: the effect of the error $F-F^{*}$ is apparent. If $F^{*}$ is evaluated numerically from its initial value-body force definition one should be careful to maintain errors smaller than those due to the interpolation scheme. Since the Green's function has strong variation near the backward characteristic surface of the receiver point, the errors from this portion of the integral are dominant. The order of $\epsilon_{j}^{n}$ is then determined by the local order of accuracy, the order of accuracy of the interpolation scheme. For interpolation scheme $D_{0}$, defined in equations (21), (23), and (24)

$$
\begin{gathered}
u-u^{*}=\mathscr{O}\left(L \frac{\partial u}{\partial s}\right)+\mathscr{O}\left(\Delta t^{2} \frac{\partial^{2}}{\partial t^{2}} u\right) \\
\tau-\tau^{*}=\mathscr{O}\left(L \frac{\partial \tau}{\partial s}\right)+\mathscr{O}\left(\Delta t \frac{\partial \tau}{\partial t}\right)
\end{gathered}
$$

where $s$ is an arc length along $\partial B$ and $L$ is the spatial discretization length. Therefore

$$
\epsilon_{j}^{n}=\mathscr{O}(L)+\mathscr{O}(\Delta t)
$$

for the interpolation method in use in Section IV.

The relation (C.3) shows that the discretization method is consistent in a conventional sense for any reasonable interpolators. To carry the analysis further some specializations are necessary. If the elastic body is the half plane $y>y_{1}$ equation (C.1) has the form

$$
u(x, t)=\frac{1}{\pi \mu} \int_{t_{0}=0}^{\infty} \int_{x_{0}=-\infty}^{\infty} \frac{H\left(t-t_{0}-\left|x-x_{0}\right| / \beta\right)}{\left[\left(t-t_{0}\right)^{2}-\left(x-x_{0}\right)^{2} / \beta^{2}\right]^{1 / 2}} \tau\left(x_{0}, t_{0}\right) d x_{0} d t_{0}+2 F(x, t) .
$$

(The argument $y_{1}$ has been dropped.) The Fourier transform with respect to $x$ of this equation is

$$
\hat{u}(k, t)=\frac{\beta}{\mu} \int_{0}^{t} J_{0}\left[k \beta\left(t-t_{0}\right)\right] \hat{\tau}\left(k, t_{0}\right) d t_{0}+2 \hat{F}(k, t)
$$

$J_{0}$ is the Bessel function of zeroth order (Gradshteyn and Ryzhik, 1965). The discretized version is the same with $\hat{u}, \hat{\tau}$, and $\hat{F}$ replaced by their approximations $\hat{u}^{*}$, $\hat{\tau}^{*}$, and $\hat{F}^{*}$. A notable feature of this equation is that since

$$
J_{0}\left(k \beta t_{0}\right) \rightarrow\left(\frac{2}{\pi k \beta t_{0}}\right)^{1 / 2} \cos \left(k \beta t_{0}-\pi / 4\right), k \beta t_{0} \gg 1 .
$$


The integral is insensitive to wave number components of $\hat{\tau}\left(k, t_{0}\right)$ for whenever $\hat{\tau}(k, t)$ is well behaved as $|k| \rightarrow \infty$. As a result the large wave number errors in $\hat{\tau}^{*}$ do not affect the accuracy of the integral after a sufficiently large number of time steps. This result is, of course, just a restatement of the properties of the medium and it is expected to hold for most boundaries. Similar results are found when the high-frequency effects in displacement integrals are examined.

Equation (C.5) is in a form suitable for stability analysis. If $\hat{\tau}^{*}$ and $\hat{F}^{*}$ are specified and $\hat{u}^{*}$ is to be calculated, it is clearly true that the iterative process is stable for a flat boundary. If $\hat{u}^{*}$ is specified and $\hat{\tau}^{*}$ is to be evaluated, there is a possibility of instability. Consider the equation for $k=0$. Let

$$
\bar{u}(t)=\int_{-\infty}^{\infty} u(x, t) d x
$$

From (C.5) we obtain

$$
\bar{u}(t)=\frac{\beta}{\mu} \int_{0}^{t} \bar{\tau}\left(t_{0}\right) d t_{0}+2 \bar{F}(t)
$$

the solution for $\bar{\tau}$ is

$$
\bar{\tau}(t)=\frac{\mu}{\beta} \frac{\partial}{\partial t}[\bar{u}(t)-2 \bar{F}(t)] .
$$

The discretized version of (C.6) for interpolators $D_{0}$ is

$$
\overline{\mathrm{u}}^{n}=\frac{\beta \Delta t}{\mu} \sum_{1}^{n} \bar{\tau}^{m}+2 \bar{F}^{n}
$$

where

$$
\bar{u}^{n}=L \sum_{j=-\infty}^{\infty} u_{j}^{n}
$$

(This relation can be deduced directly from equations (26) and (27) by summation over $j$.) The analog of $(\mathrm{C} .6 \mathrm{a})$ is readily found from (C.7) to be accurate to order $\Delta t$

$$
\bar{\tau}^{n}=\frac{\mu}{\beta}\left(\frac{\bar{u}^{n}-\bar{u}^{n-1}}{\Delta t}-2 \frac{\bar{F}^{n}-\bar{F}^{n-1}}{\Delta t}\right)
$$

$\bar{\tau}^{n}$ is dependent only on the data at time levels $n$ and $n-1$, so this particular scheme is stable for sufficiently small wave numbers. If one were to use a different interpolation method for $\tau^{*}$ the result would not necessarily be the same. For example, if $T^{n}(t)$ were linearly varying in time, as $U^{n}$ is in (24), instead of being piecewise constant, as it is in (23), we would have

$$
D G_{i j}^{m}=\frac{\beta \Delta t}{\pi \mu}\left(E_{m}^{+1}+E_{m}^{-1}-2\right)\left(E_{k}^{+1 / 2}-E_{k}^{-1 / 2}\right) m^{2} H(m) g_{1}\left(\frac{k}{Q m}\right)
$$


where

$$
g_{1}(\eta)= \begin{cases}\eta \ell n\left[|\eta|^{-1}+\left(\eta^{-2}-1\right)^{1 / 2}\right]+\frac{1}{2} \sin ^{-1}(\eta) \\ -\eta|\eta| \frac{1}{2}\left[\eta^{-2}-1\right]^{1 / 2}, & |\eta| \leqq 1 \\ \frac{\pi}{4}, & |\eta| \geqq 1\end{cases}
$$

and $k=|i-j|$.

For this interpolator

The solution for $\bar{\tau}^{n}$ is

$$
\bar{u}^{n}=\frac{\beta \Delta t}{2 \mu}\left(\bar{\tau}^{n}+2 \sum_{1}^{n-1} \bar{\tau}^{m}\right)+2 \bar{F}^{n} .
$$

$$
\bar{\tau}^{n}=-\bar{\tau}^{n-1}+\frac{2 \mu}{\beta}\left\{\frac{\bar{u}^{n}-\bar{u}^{n-1}}{\Delta t}-2 \frac{\bar{F}^{n}-\bar{F}^{n-1}}{\Delta t}\right\}
$$

or

$$
\bar{\tau}^{n}=\frac{2 \mu}{\beta \Delta t}\left\{\bar{u}^{n}-2 \bar{F}^{n}+(-1)^{n} 2 \sum_{m=1}^{n-1}(-1)^{m}\left(\bar{u}^{m}-2 \bar{F}^{m}\right)\right\}
$$

This solution for $\bar{\tau}^{n}$ is clearly unacceptable; from (C.10) it is readily seen that arbitrarily large errors of $\bar{\tau}^{n}$ may be made to persist for all time, for example by setting

$$
\bar{u}^{1}-2 \bar{F}^{1}=A, \bar{u}^{m}-2 \bar{F}^{m}=0, m>1 .
$$

Then $\bar{\tau}^{n}=(-1)^{n-1} \frac{4 \mu}{\beta \Delta t} A, n>1$. Equation (C.9) more closely represents the actual numerical iteration, so in terms of machine manipulations the solution would be

$$
\bar{\tau}^{n}=(-1)^{n-1} 2 \bar{\tau}^{1}, n>1 \text {. }
$$

This process is marginally stable in theory and has been found to be unstable in practice. The type of divergence arising is global; one finds that after many time steps

$$
\tau_{j}^{n}=C_{j}(-1)^{n}(1+a)^{n}, a>0
$$

where the constants $C_{j}$ are all of the same sign. For a bonded interface the two interpolation schemes give identical results when solved for $\bar{u}^{n}$ but the linear traction interpolators again produce divergent tractions.

A complete stability analysis of the approximated version of (C.5) should include all wave numbers $k$. This cannot be carried out analytically due to the appearance of the special function $J_{0}$. It is possible, however, to argue that the type of divergence 
shown above is typical and detectable by the zero wave number analysis. First note that

$$
G\left(\mathbf{r}, t, \mathbf{r}_{0}, t_{0}\right) \geqq 0
$$

A realistic discrete kernel $D G$ should have $D G_{i j}{ }^{n} \geqq 0$ for small $n$. In the iterative solution for $\tau^{*}$, then, $\tau_{j}^{n}$ will depend on an average over $i$ of $\tau_{i}^{n-1}, \tau_{i}^{n-2}, i=j, j \pm 1$, $j \pm 2$. That is, there is no numerical differentiation along the boundary. If $u_{j}^{n}-2 F_{j}^{n}$ is smoothly varying with respect to $j$, any large wave number oscillations of the tractions will be smoothed out rapidly in the iterative process. Thus, it seems unlikely that divergence of the form $\tau_{j}^{n}=C_{j}(-1)^{j+n}(1+a)^{n}$ could occur, and any instability would be caused by badly approximating the derivative with respect to time in (C.6a).

It is possible to demonstrate that $D_{0}$ produces a convergent algorithm for the two fundamental boundary value problems of the half plane. For the first problem, in which the traction is specified, it is sufficient to note that the error estimates (C.3) and (C.3a) give a bound for $u^{*}-u$ proportional to $\Delta t$ and $L$. Therefore, if $\tau(x, t)$ is sufficiently differentiable $u^{*}(x, t) \rightarrow u(x, t)$ as $L \rightarrow 0$ with $\beta \Delta t / L$ held constant.

The other fundamental problem is one in which $u(x, t)$ is specified. Due to the singularities of the Green's function, manipulation of (C.4) presents serious difficulties. Instead, the wave number domain expression (C.5) will be used. The transform of $\tau^{*}(x, t)$ is given by

$$
\hat{\tau}^{*}(k, t)=\frac{\sin (k L / 2)}{(k L / 2)} \sum_{j} L e^{i k x j} \tau_{j}^{n}, t_{n}>t>t_{n-1} .
$$

The discussion here will assume $k L / 2<1$ to permit an interpretation of $\hat{\tau}^{*}$ and $\hat{u}^{*}$, which is given by a similar formula with time interpolation the same as in $D_{0}$. For explicit time stepping the time step size is restricted to $\beta \Delta t \leqq L / 2$ and hence $k \beta \Delta t<1$.

By differentiating (C.5) one obtains for the exact solution

$$
\frac{\mu}{\beta} \frac{\partial}{\partial t} \hat{u}(k, t)=\hat{\tau}(k, t)+\int_{q=0}^{t} \tau(k, q) \frac{\partial}{\partial t} J_{0}(k \beta(t-q)) d q .
$$

The time stepping version of (C.5) is

$$
\frac{\mu}{\beta} \hat{u}^{n}=\sum_{m=1}^{n} J_{0}^{n-m} \hat{\tau}^{m}
$$

where

$$
\begin{aligned}
& \hat{u}^{n}=\hat{u}^{*}\left(k, t_{n}\right)=\hat{u}\left(k, t_{n}\right) \\
& \hat{\tau}^{m}=\hat{\tau}^{*}\left(k, t_{m}-\Delta t / 2\right)
\end{aligned}
$$

and

$$
J_{0}^{n-m}=\int_{t_{m-1}}^{t_{m}} J_{0}\left(k \beta\left(t_{n}-q\right)\right) d q
$$


Differencing (C.12) gives

$$
\frac{\mu}{\beta} \frac{1}{J_{0}{ }^{0}}\left(\hat{u}^{n}-\hat{u}^{n-1}\right)=\hat{\tau}^{n}+\sum_{m=1}^{n-1} \frac{1}{J_{0}{ }^{0}}\left(J^{n-m}-J^{n-1-m}\right) \hat{\tau}^{m} .
$$

Subtracting this from (C.11) gives an expression for the error as it would be produced in the time stepping procedure

$$
\begin{aligned}
\hat{\tau}\left(k, t_{n}-\Delta t / 2\right)-\hat{\tau}^{n}= & \frac{\mu}{\beta}\left\{\left.\frac{\partial}{\partial t} \hat{u}(k, t)\right|_{t_{n}-\Delta t / 2}-\frac{1}{J_{0}{ }^{0}}\left(\hat{u}^{n}-\hat{u}^{n-1}\right)\right\} \\
& +k \beta \int_{q=0}^{t_{n}-\Delta t / 2} \hat{\tau}(k, q) J_{1}\left(k\left(t_{n}-\Delta t / 2-q\right)\right) d q \\
& +\sum_{m=1}^{n-1} \hat{\tau}^{m} \frac{1}{J_{0}{ }^{0}}\left(J^{m-m}-J^{n-m-1}\right) .
\end{aligned}
$$

Since

$$
J_{0}{ }^{0}=\Delta t\left\{1-\left(\frac{k \beta \Delta t}{2}\right)^{2} \frac{1}{3}+\ldots+\mathscr{O}(h \beta \Delta t)^{2 n}\right\}
$$

and

$$
J^{n-m}-J^{n-1-m}=-k \beta \Delta t \int_{t_{m-1}}^{t_{m}} J_{1}\left[k \beta\left(t_{n}-q\right)\right] d q+\mathscr{O}(k \beta \Delta t)^{2}
$$

This can be reduced to

$$
\begin{aligned}
\hat{\tau}\left(k, t_{n}-\Delta t / 2\right)-\hat{\tau}^{n}= & \frac{\mu}{\beta}\left[\left.\left(1-\frac{\Delta t}{J_{0}^{0}}\right) \frac{\partial}{\partial t} u(k, t)\right|_{t_{n}-\Delta t / 2}-\mathcal{O} \frac{\Delta t^{3}}{J_{0}^{0}} \frac{\partial^{3}}{\partial t^{3}} \hat{u}\right] \\
& +k \beta \int_{0}^{t_{n-1}}\left\{\left[\hat{\tau}(k, q)-\hat{\tau}^{*}(k, q)\right] J_{1}\left[k \beta\left(t_{n}-q\right)\right]+\mathscr{O}\left(\hat{\tau}^{*} k \beta \Delta t\right)\right\} d q .
\end{aligned}
$$

For any fixed value of $k$, as $L \rightarrow 0$ with $\beta \Delta t / L$ held constant, the error inside the integral arising from the approximation of $J_{1}$ is dominant and of order $\Delta t$. Therefore, by induction over time step $n$

$$
\hat{\tau}\left(k, t_{n}-\Delta t / 2\right)-\hat{\tau}^{n}=\mathscr{O}\left(k^{2} \beta^{2} \Delta t\right)
$$

for all time steps; as $\Delta t \rightarrow 0, \hat{\tau}^{*}(k, t) \rightarrow \hat{\tau}(k, t)$. The general behavior of the error for fixed $\Delta t$ and $L$ can also be seen to be proportional to the wave number, i.e., errors at wavelength $\lambda$ will be proportional to $L / \lambda$.

It should be noted that, as in any numerical calculation, convergence depends on the differentiability of the boundary values and source terms. This has important consequences when one is approximating a mixed-type boundary value problem 
because stress discontinuities commonly arise at points where the type of boundary condition changes. It is possible to demonstrate that many BIE methods will not converge for these problems.

A short digression seems in order here regarding equation (C.6). This relation has a particularly simple physical interpretation. The one-dimensional wave equation may be factored in the form

$$
\left(\frac{1}{\beta} \frac{\partial}{\partial t}+\frac{\partial}{\partial y}\right)\left(\frac{1}{\beta} \frac{\partial}{\partial t}-\frac{\partial}{\partial y}\right) u(y, t)=g(y, t), t>0, y \geqq y_{1} .
$$

The first factor corresponds to the characteristics of waves traveling in the positive $y$ direction and is very similar in form to the homogeneous part of (C.6), which may be written as

$$
\left.\left(\frac{1}{\beta} \frac{\partial}{\partial t}+\frac{\partial}{\partial y}\right) \bar{u}(y, t)\right|_{y=y_{1}}=\left.\frac{2}{\beta} \frac{\partial}{\partial t} \bar{F}(y, t)\right|_{y=y_{1}}
$$

Thus (C.6) is an equation for waves traveling from $y=y_{1}$ into $y>y_{1}$. The inhomogeneous term $\vec{F}$ is due to sources in the region $y>y_{1}$ and they must propagate along characteristics traveling in the negative $y$ direction at $y=y_{1}$. If $g=0$ near $y=y_{1}$, the solutions of (C.14) there are of the form

$$
u(y, t)=u^{+}(\beta t-y)+u^{-}(\beta t+y)
$$

and obey the relation

$$
\left(\frac{1}{\beta} \frac{\partial}{\partial t}+\frac{\partial}{\partial y}\right) u=\frac{2}{\beta} \frac{\partial}{\partial t} u^{-}(\beta t+y)
$$

which compares term by term with (C.15). Analogies of this sort may be seen for the general BIE, equation (9), by considering local portions of space-time near $\partial B$.

\section{ReFERENCES}

Archambeau, C. B. and J. B. Minster (1977). Dynamics in prestressed media with moving phase boundaries; a continuum theory of failure in solids, Geophys. J. 52, 65-96.

Burridge, R. (1969). The numerical solution of certain integral equations with non-integrable kernels arising in the theory of crack propagation and elastic wave diffraction, Proc. Roy. Soc. London A, 265, 353-381.

Courant, R. and D. Hilbert (1966). Methods of Mathematical Physics, Vol. II, Ch. VI, Interscience Publishers, New York.

Cruse, T. A. (1968). A direct formulation and numerical solution of the general transient elastodynamic problem I, J. Math. Anal. Appl. 22, 341-355.

Cruse, T. A. and F. J. Rizzo (1975). Boundary-integral equation method: Computational applications in applied mechanics, Am. Soc. Mech. Eng., AMD, 11, New York.

Das, S. (1976). A numerical study of rupture propagation and earthquake source mechanism, Thesis, Massachusetts Institute of Technology.

Das, S. and K. Aki (1977). Numerical study of two-dimensional spontaneous rupture propagation, Geophys. J. 50, 643-668.

de Hoop, A. T. (1958). Representation theorems for the displacement in an elastic solid and their application to elastodynamic diffraction theory, Thesis, Technische Hogeschool Delft, Delft, The Netherlands. 
Friedman, M. B. and R. Shaw (1962). Diffraction of pulses by cylindrical obstacles of arbitrary cross section, Trans. Am. Soc. Mech. Eng., JiAppl. Mech., Ser. E, 29, 40-46.

Gradshteyn, I. S. and I. M. Ryzhik (1965). Table of Integrals, Series, and Products, p. 953, 8.411.8, Academic Press, New York.

Kellogg, O. D. (1929). Foundations of potential theory, Ch. VI, Dover Publications, New York. Reprinted 1953.

Kupradze, V. D. (1963). Dynamical Problems in Elasticity, p. 86, North-Holland Publishing Co., Amsterdam.

Love, A. E. H. (1944). A treatise on the mathematical theory of elasticity, 4th ed., Dover Publications, New York.

Mitzner, K. M. (1967). Numerical solution for transient scattering from a hard surface of arbitrary shaperetarded potential technique, J. Acoust. Soc. Am. 42, 391-397.

Mow, C.-C. and Y.-H Pao (1971). The diffraction of elastic waves and dynamic stress concentrations, Rand Corporation, Santa Monica, Ca.

Wheeler, L. T. and E. Sternberg (1968). Some theorems in classical elastodynamics, Arch. Rat. Mech. Anal. 31, 51-90.

SeIsmological Laboratory

CALIFORNIA INSTITUTE OF TechNology

Pasadena, California 91125 (D.M.C. \& J.B.M.)

Contribution No. 3008

Manuscript received February 16, 1978
HEBREW UNIVERSITY OF JERUSALEM

JERUSALEM, ISRAEL (D.D.K.) 